\title{
Odor quality: Discrimination versus free and cued identification
}

\author{
RENÉ A. DE WIJK and WILLIAM S. CAIN \\ John B. Pierce Laboratory and Yale University, New Haven, Connecticut
}

\begin{abstract}
Eighty-two adults, ranging in age from young to elderly, performed odor-quality discrimination and both free and cued identification on six odorants presented at two intensity levels. The odorants simulated the real-world substances banana, licorice, cherry/almond, wintergreen, clove, and lemon. Performance on all three tasks declined with age, but improved with stimulus intensity. Performance at discrimination benefited from the mere availability of the six names during testing. Performance in cued identification far exceeded that in free identification and, for young and middle-aged adults, fell close to that for discrimination. For elderly adults, however, performance in cued identification fell substantially below that in discrimination. Although not entirely free of cognitive influences, discrimination seems to offer particularly clear resolution of alterations in olfactory functioning.
\end{abstract}

The assessment of olfaction in both experimental and clinical studies often involves determination of ability to identify odors, ability to detect an odor, or both. One or both tasks appear, for instance, in standardized tests of olfaction, such as the University of Pennsylvania Smell Identification Test (UPSIT) and the Connecticut Chemosensory Clinical Research Center (CCCRC) test (Cain, Gent, Goodspeed, \& Leonard, 1988; Doty, Shaman, \& Dann, 1984b). Tests of detection and tests of identification reflect somewhat different underlying processes. Whereas detection reflects principally a sensory process, identification reflects both sensory and cognitive processes (see Cain \& Gent, 1986). Nevertheless, in people with normal cognition the tests correlate strongly; the correlation coefficient has equaled .8 to .9 in mixed samples of normosmics and people with hyposmia and anosmia (Cain \& Rabin, 1989; Doty et al., 1984b).

The high correlation between a measure of sensitivity (detection) and the ability to identify odors essentially validates the use of identification to assess hyposmia and anosmia, since sensitivity is the criterion by which such conditions are defined (see Cain \& Rabin, 1989). Between the tasks of detection and identification lies a linking process of quality discrimination. Hence, in the hyposmic subject poor olfactory sensitivity presumably impairs quality discrimination, which in turn reduces identifiability. Despite its pivotal position in the hierarchy of olfactory talents, quality discrimination has received little direct at-

This research was supported by Grant DC 00284 from the National Institutes of Health. We thank Nicole Pollard for able assistance. Correspondence should be addressed to William S. Cain, Department of Surgery, University of California, San Diego, San Diego, CA 92103.

-Accepted by previous editor, Charles W. Eriksen tention. In studies where normals and epilepsy patients have participated in both quality discrimination and identification, among other tasks, correlations have fallen in the range .5 to .8 (e.g., Eskenazi, Cain, Novelly, \& Friend, 1983).

Quality discrimination takes on particular importance in cases of olfactory loss from nondementing lesions of the central nervous system. Patients with epilepsy, for example, have shown clinically normal absolute sensitivity but poor ability to discriminate quality (Eskenazi et al., 1983; Eskenazi, Cain, Novelly, \& Mattson, 1986; Martinez et al., 1993; Zatorre \& Jones-Gotman, 1991). Insofar as quality discrimination proves vulnerable to such central losses and yet makes fewer cognitive demands than identification, its potential utility seems worthy of greater attention.

The goal of the present study was to determine the relevance of several variables to performance at discrimination. The study examined the relationship between odor discrimination and intensity of stimulation and both free identification and cued identification of simulants of realworld odors. Simulants served as the stimuli because they could be matched for perceived intensities and could thereby allow systematic manipulation of this variable. In order to examine the relative merits of the three tasks in subjects who might vary in cognitive ability, the investigation used three groups of quite different agesyoung, middle-aged, and elderly. Aging is known to take a toll on both threshold and suprathreshold functioning (Cain \& Stevens, 1989; Doty et al., 1984a; Eskenazi, Cain, \& Friend, 1986; Murphy, 1983; Schiffman, Moss, \& Erickson, 1976; Stevens \& Cain, 1985; Wood \& Harkins, 1987). Moreover, it seems to take a toll on odor identification from cognitive losses, beyond any sensory loss (Schemper, Voss, \& Cain, 1981). Hence, the variable of aging provides a vehicle to examine how cogni- 
tive and sensory influences combine to alter olfactory performance.

Although the principal focus here was a comparison of the identification task and the discrimination task, results of Rabin (1988) led us to explore the top-down influence of semantic information on quality discrimination. Rabin demonstrated that teaching subjects to name test odors with veridical labels before a discrimination task improved their performance relative to that of subjects given mere exposure or the structured exposure of profiling of quality without the labels. The present study examined, in part, a less aggressive way to make the labels available to the subjects: The list of names of the odors was simply made available during the discrimination task.

\section{METHOD}

\section{Subjects}

Eighty-two subjects, between 18 and 88 years of age, participated in two sessions lasting 1 to $1.5 \mathrm{~h}$. Three groups comprised 47 young adults (18-33 years), 18 middle-aged adults (34-60 years), and 17 elderly adults (69-88 years), respectively, with approximately equal numbers of males and females per group. The young adults were employees of the Pierce Laboratory or students at Yale University; those in the middle-aged group were employees at the Pierce Laboratory or Yale University; and the elderly were members of local senior centers. The latter lived independently, professed normal health and olfaction for their age, and could travel to the laboratory for testing. In some instances they came to the laboratory and on other occasions they went to their senior centers for testing. Whereas most of those in the young and middle-aged groups had college-level education, most in the elderly group did not.

\section{Stimuli}

The test stimuli, each at two levels of intensity, were: amyl acetate, $0.10 \%$ and $1.21 \% \mathrm{v} / \mathrm{v}$ in mineral oil (banana odor); methyl chavicol coeur, $0.17 \%$ and $1.01 \%$ (licorice odor); benzaldehyde, $0.17 \%$ and $1.01 \%$ (maraschino-cherry or almond odor); methyl salicylate, $0.17 \%$ and $1.01 \%$ (wintergreen odor); eugenol, $0.17 \%$ and $1.01 \%$ (clove odor); and citral, $0.17 \%$ and $1.01 \%$ (lemon odor). Higher and lower concentrations, respectively, were approximately matched for perceived intensity across odorants. A pilot study, in which detection thresholds for amyl acetate were determined for young and elderly subjects, implied that the concentrations used in this study exceeded detection thresholds by a factor of 30 or more. Cylindrical Chromex Interflo pellets $(9 \mathrm{~mm}$ diam $\times 9 \mathrm{~mm}$ height), which absorbed $0.2-\mathrm{ml}$ quantities of solution, served as the actual sources of odor. Such pellets release odorant from their surfaces uniformly over time. Odorized pellets, two per bottle, were placed into $240-\mathrm{ml}$ polypropylene squeeze bottles with pop-up spouts (see Cain, 1989).

\section{Procedure}

The testing proceeded as follows: First, all the subjects participated in 120 trials of quality discrimination distributed over the two sessions ( 60 trials per session). After the 60 trials of discrimination in the second sesstion, approximately half the subjects $(51 \%$ of the young group, $50 \%$ of the middle-aged group, and $59 \%$ of the elderly group) participated in 12 trials of free identification. Finally, all the subjects participated in 12 trials of cued identification at the end of the second session. Those subjects who did not participate in the 12 trials of the second stage-that is, who skipped free identification-received a printed list of the six test odors ("banana," "licorice," "cherry," "cloves," "wintergreen," and "lemon") from the outset of discrimination testing, and could refer to the list throughout testing. These subjects comprised the ex- perimental group for testing the hypothesis that availability of semantic information would assist discrimination. The subjects who went through discrimination testing without the list-that is, the control group-eventually received the list of names for the cuedidentification task.

The stimulus set for discrimination was made up of 120 pairs. Sixty pairs comprised all combinations of the different qualities and intensities (low-low, low-high, high-low, and high-high) and 60 comprised the pairs of the same quality, irrespective of intensity. Since combinations with different qualities outnumbered those with the same quality (60 vs. 24 ), pairs of a single quality were presented in duplicate or triplicate in order to equalize presentations of same and different pairs. Time between trials averaged $45 \mathrm{sec}$. Order of presentation varied irregularly across subjects.

On the trials of the discrimination task, the subjects successively squeezed each bottle of a pair just below their nostrils and sought to decide whether the bottles smelled the same or different in quality without respect to perceived intensity. Those participants who had the printed names (cues) available received the additional instruction that their performance might benefit from reference to the names. In the twelve trials of the free-identification task, the subjects received one bottle at a time, squeezed it just below their nostrils, and sought to identify its odor. Thereafter, both those subjects who had previously received the list and those who had not could refer to it as they sought to identify each odor at each intensity.

\section{Scoring}

The nonparametric index $A^{\prime}$ was used to summarize each subject's performance in discrimination (Gescheider, 1984; Pollack \& Norman, 1964). The index, which translates into the familiar term proportion correct in a two-alternative forced-choice task, has the following formula:

$$
\begin{aligned}
A^{\prime}= & 1 / 2+ \\
& \frac{[p(\text { hit })-p(\text { false alarm })][1+p(\text { hit })-p(\text { false alarm })]}{[4 \times p(\text { hit })][1-p(\text { false alarm })]},
\end{aligned}
$$

where $p$ (hit) represents proportion of hits, $p$ (false alarms) represents proportion of false alarms, and so on. Hits and misses were scored, respectively, when a subject responded correctly and incorrectly to two odors with the same quality. Correct rejections and false alarms were scored, respectively, when a subject responded correctly and incorrectly to odors with different qualities.

For analysis of discrimination performance by item, percent correct discrimination was used; when subjects correctly judged two odors as different, they were credited with a correct response for each odor, and when they correctly judged two odors as the same, they were credited with a correct response for that odorant. For scoring of identification performance, only veridical labels or reasonable equivalents were scored as correct; in the case of the stimulus licorice, for example, the names licorice and anise were counted as equivalent.

\section{RESULTS}

\section{Analysis by Subject}

Performance $\left(A^{\prime}\right)$ in discrimination averaged 0.90 and declined from $.95 \pm .01(S E)$ through $.89 \pm .02$ to $.78 \pm .03$ for young, middle-aged, and elderly subjects, respectively $[F(2,81)=32.0, p=.0001]$. Post hoc tests (NewmanKeuls, $p<.05$ ) revealed that the young outperformed both other groups and that the middle-aged outperformed the elderly (Figure 1). Aging led to both reduced hit rate and increased false-alarm rate (see Figure 2).

The subjects discriminated better at the higher level of intensity $\left(A^{\prime}=.93 \pm .01[S E]\right)$ than at the lower level 
Higher vs Lower Intensity

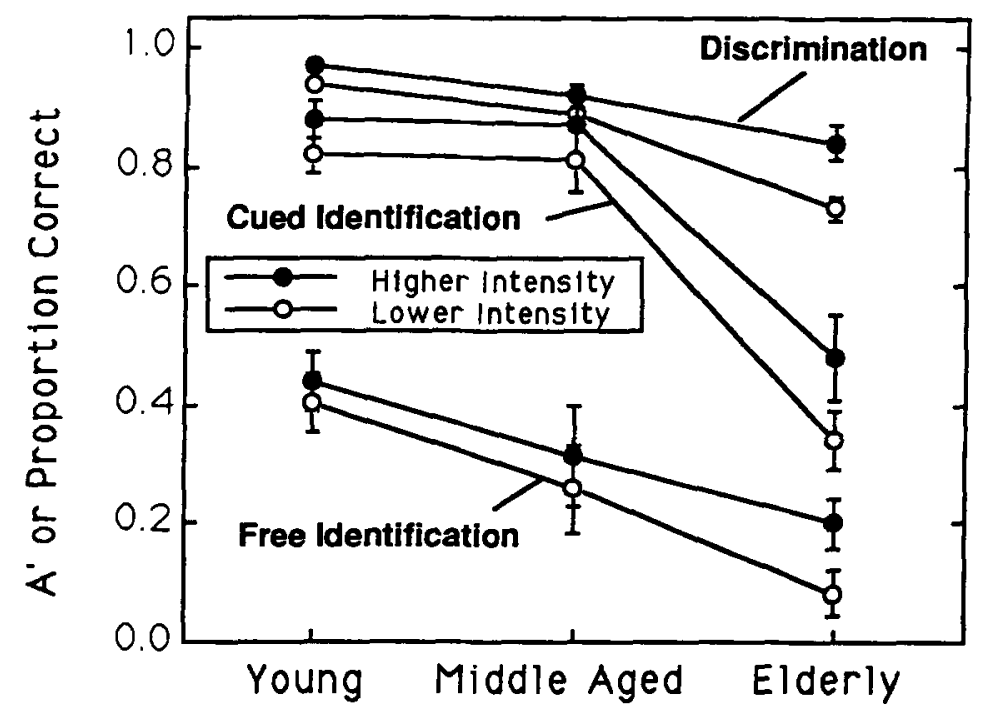

Figure 1. Performance $( \pm S E)$ in quality discrimination $\left(A^{\prime}\right)$ and in cued and free identification (proportion correct), at two intensity levels, versus age.

$\left(A^{\prime}=.88 \pm .01\right)$ or at mixed levels $\left(A^{\prime}=.89 \pm .01\right)$ $[F(2,162)=18.3, p<.001]$ (Figure 1). Discrimination at the lower level proved relatively more difficult for the elderly than for the others [age $x$ intensity interaction: $F(1,70)=10.2, p<.001]$.

The availability of odor names enhanced discrimination $[F(1,76)=4.2, p=.04]$ (Figure 3 ). The names proved significantly more effective at the higher level $[F(1,76)=5.7, p=.02]$ and at the mixed levels $[F(1,76)=5.5, p=.02]$. A significant interaction of label $\times$ age $\times$ intensity $[F(4,152)=2.7, p=.03]$ arose principally from a loss of an advantage from the names at the lower level for the middle-aged and elderly subjects.

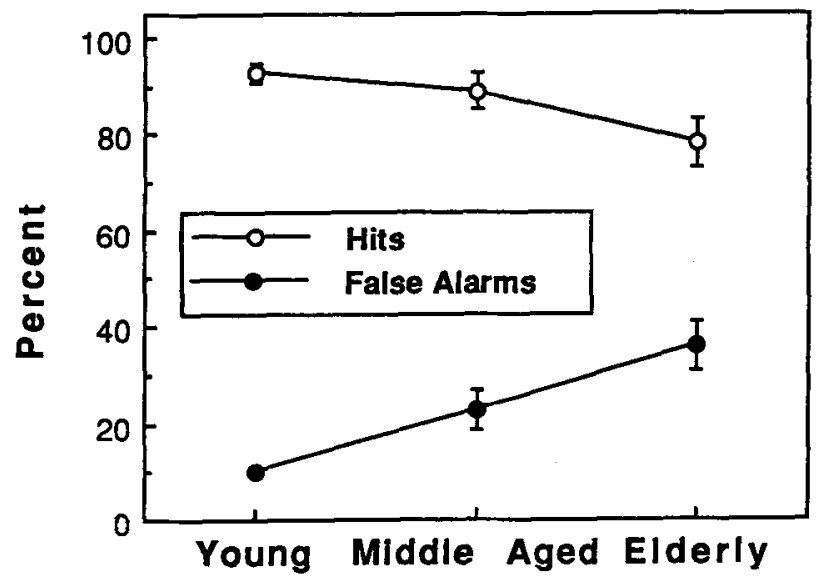

Figure 2. Percentages $( \pm S E)$ of hits and false alarms versus age in quality discrimination.
Free identification averaged $33 \%$ correct and also varied significantly with age $[F(2,40)=5.2, p=.01]$ (Figure 1). The relatively low average performance presumably arose from the use of simulants (cf. Cain, 1979; Stevens, Cain, \& Demarque, 1990). As with discrimination, the subjects identified the higher level better $[F(1,40)=9.1, p=.004]$. Cued identification, which averaged $76 \%$ correct, fell below performance in discrimination and also declined with age $[F(2,79)=34.6, p<$ $.01]$ (Figure 1). The higher level also allowed easier cued identification $[F(1,80)=13.4, p=.0005]$.

The relationship between age and performance in the various tasks revealed itself additionally in significant negative correlations between age and, respectively, discrimination $(r=-.64, p<.01)$, free identification $(r=$ $-.49, p<.01)$, and cued identification $(r=-.63, p<$ $.01)$. The association between aging and discrimination remained significant even with cued identification as a covariate $[F(2,78)=11.5, p=.0001]$. Deterioration of discrimination with age therefore seemed independent of any loss of ability to identify.

Discrimination correlated significantly with identification $(r=.51$ and $r=.59, p<.01$, for free and cued identification, respectively). Discrimination also correlated well with the consistency with which subjects, in cued identification, chose the same name, either correct or incorrect, at the two intensity levels for each test odor $(r=.61, p<.01)$. Consistency of naming also correlated well with age $(r=-.63, p<.01)$.

\section{Analysis by Odorant}

Performance in free identification varied considerably from one stimulus to another. Those in the young group 

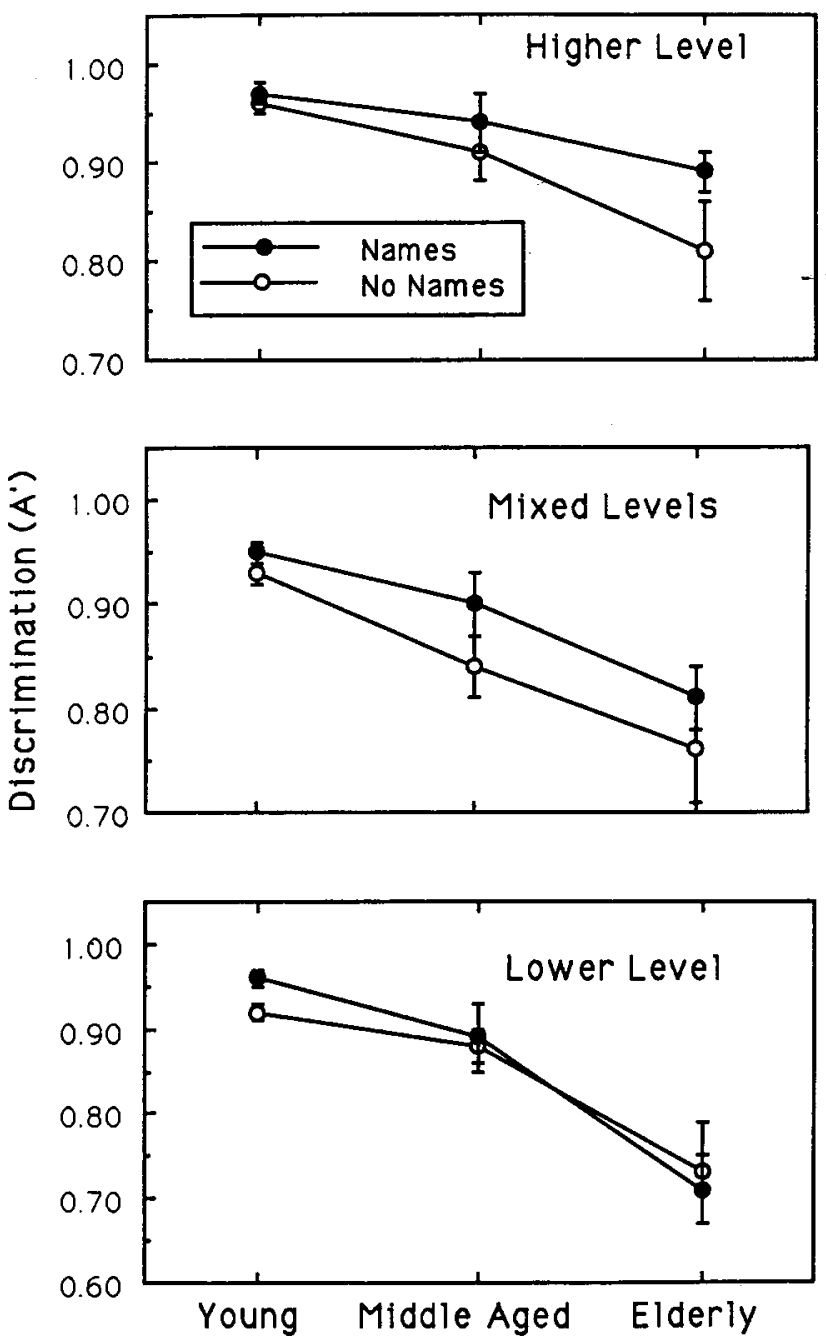

Figure 3. Influence of the availability of odor names on discrimination $\left(A^{\prime} \pm S E\right)$ at the higher, lower, and mixed levels of intensity.

generally exhibited the least variation, though they still varied approximately threefold from the stimulus they identified best-licorice-to the one they identified worst-wintergreen (Figure 4). Those in the middle-aged group showed a similar disparity, but identified banana best and wintergreen worst. Those in the elderly group also identified licorice best and, at the low intensity, never identified wintergreen and lemon. The profile for the three groups across odorants showed surprisingly little consistency. For instance, the elderly subjects sometimes performed about the same as the middle-aged subjects on an item and sometimes performed much worse. Correlations of the profile of performance across the 12 stimuli (6 odorants $\times 2$ levels) for all six pairs of the three age groups averaged .36 , and only one achieved significance (young vs. elderly: $r=.60$ ).

Some simulants mimicked their real-world counterparts better than others. In order to identify simulants correctly, a person might need to "bend" qualities into their appropriate mental templates. Cued by names, subjects can often accomplish this surprisingly well. With such aid, those in the young and middle-aged groups overcame some of their weakness regarding wintergreen (Figure 4). The middle-aged subjects also made up considerable ground with licorice, cherry, and cloves. With cuing, differences due to level also tended to shrink, particularly for the young and middle-aged subjects. Correlations of the profile of performance across the 12 stimuli for all six pairs of the three age groups here averaged .45 , again with only one significant coefficient (middle-aged vs. elderly: $r=.70$ ).

Discrimination displayed much greater uniformity across stimuli (Figure 4). Performance of those in the young group varied within about $9 \%$, both at the lower and higher levels. Corresponding variation in performance in the middle-aged group equaled $38 \%$ and $27 \%$, and that 

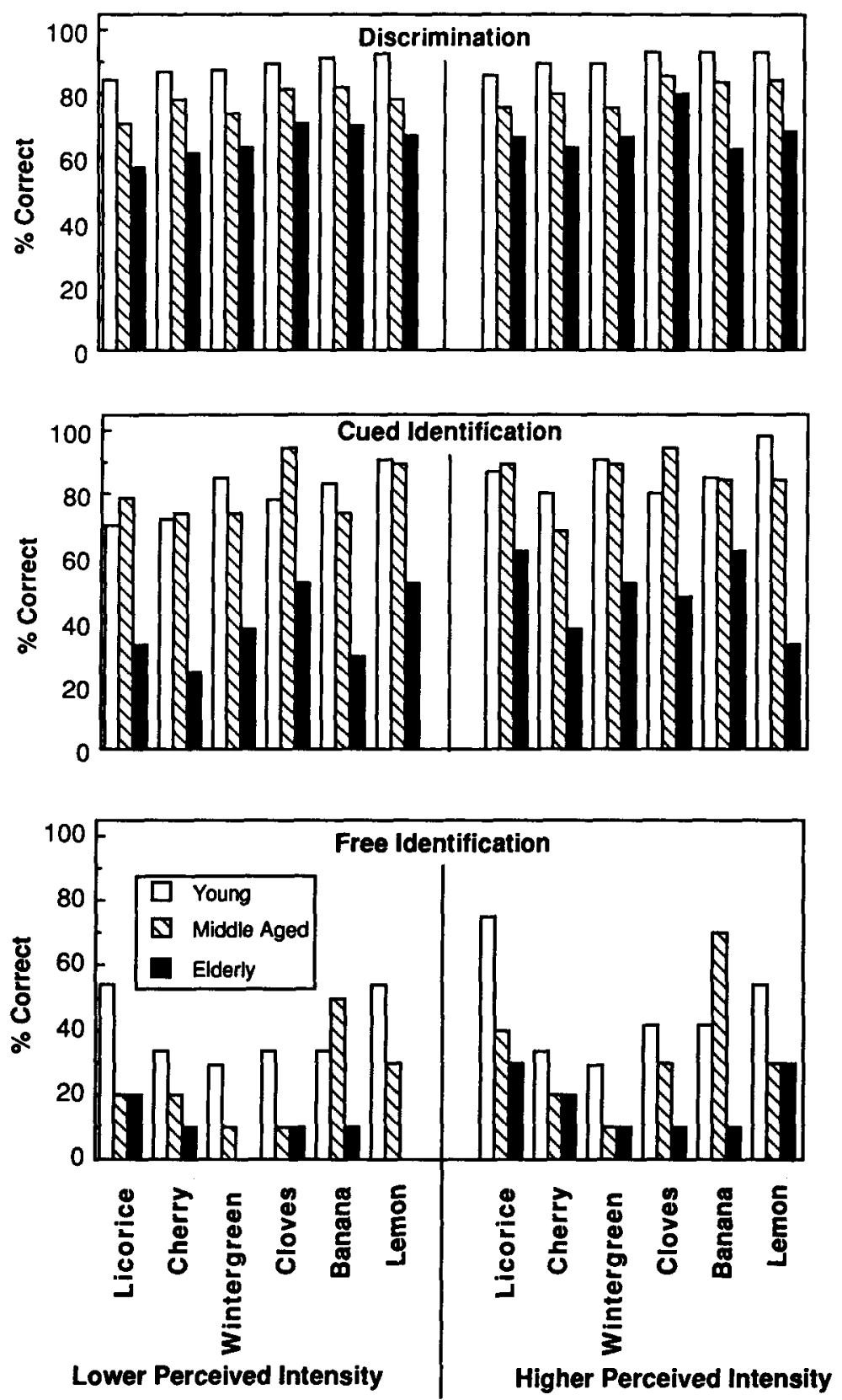

Figure 4. Performance (percent correct) per odorant in discrimination and in cued and free identification, at lower and higher intensities.

in the elderly group equaled $25 \%$ and $28 \%$. Correlations of the profile of performance across the 12 stimuli were significant in all six cases and ranged from $.60(p<.05)$ to $.82(p<.01)$, an outcome that also reflects the less idiosyncratic nature of the performance in discrimination.

Figure 5 illustrates that, for all six odors, cued identification matched discrimination rather well for the young and middle-aged groups. For the elderly group, cued identification fell rather uniformly below discrimination.

\section{DISCUSSION}

All three of the tasks presented to the subjects showed sensitivity to variables known or suspected to influence olfactory performance. Whereas age impaired performance considerably, intensity enhanced it, though only modestly. Nevertheless, the more difficult the task (e.g., free identification) or the more disadvantaged the subjects (e.g., the elderly), the greater the proportional enhanc- 

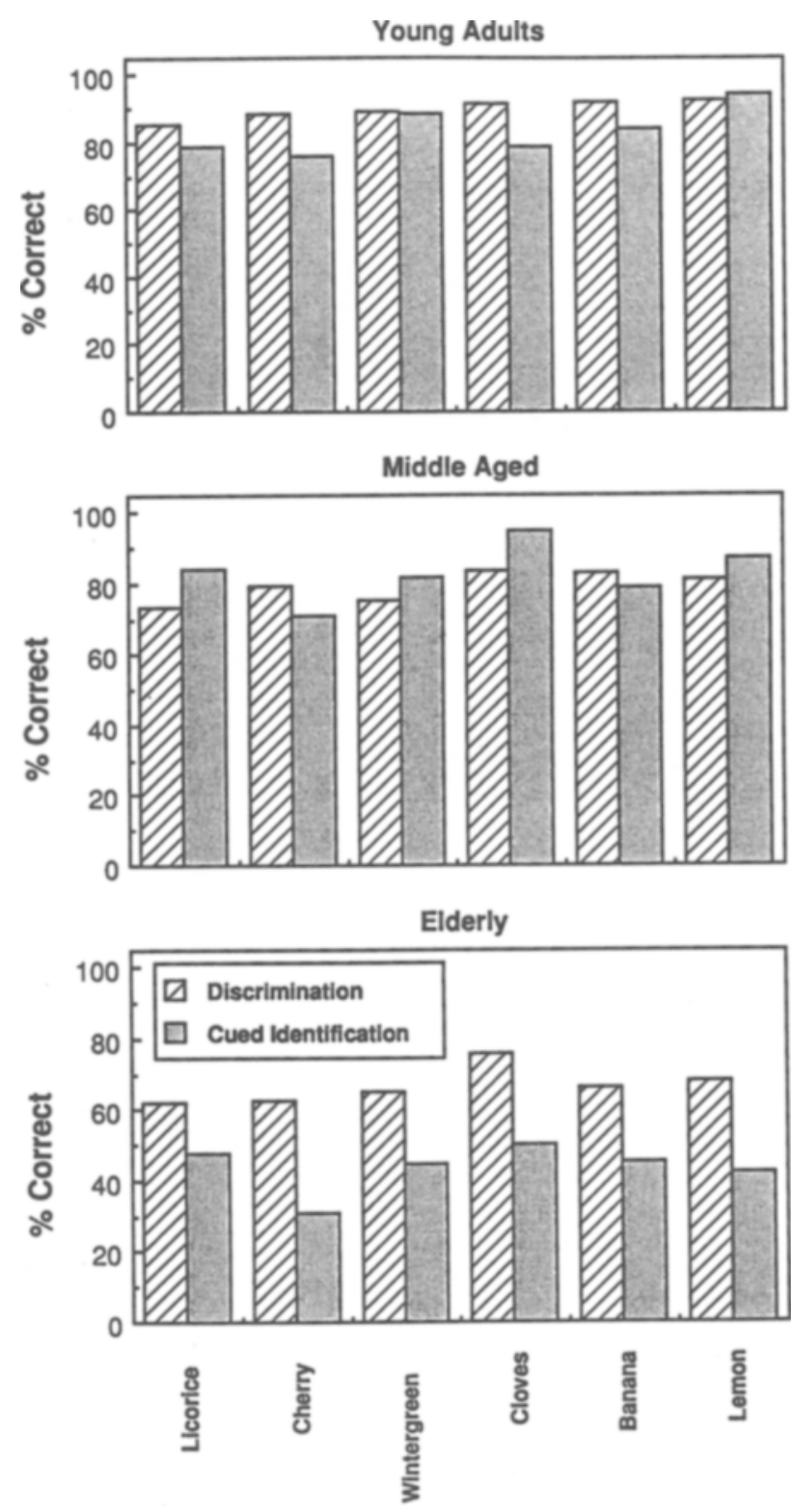

Figure 5. Discrimination versus cued identification (percent correct) per odorant.

ing effect of intensity. The availability of odor names enhanced discrimination, just as Rabin's (1988) results seemed to anticipate. As Rabin was the first to show, semantic information about odor stimuli has a facilitatory effect on discrimination, the most basic qualitative judgment of all. In his investigation, the subjects received rather intensive paired-associate training with the labels and odors prior to the discrimination task. In the present investigation, the subjects simply had the names available, but even this still sharpened discrimination. Presumably, semantic information about odors becomes part of a feedback loop that may enhance both discrimination and identification. The better a person can discriminate between stimuli, the easier it may be to apply different names to them, but the availability of different names (e.g., cloves vs. ginger), with their attendant meanings, can make it easier to perceive these discriminably different stimuli as categorically different.

The discrimination task offered particular uniformity of outcome across stimuli. In the comparison of every odor with every other, none looked very different from any other. The discrimination test would accordingly seem to pose only a rather small chance that the outcome for any particular subject will depend upon a fortuitous choice of stimuli.

Although previous results had implied that the elderly 
might find free identification difficult for cognitive as well as for sensory reasons, the same possibility had not yet been noted for cued identification. The present investigation implied some effect. Cued identification produced largely the same outcome as discrimination did in the young and middle-aged groups. With both measures corrected for chance, cued identification (chance level $=.17$ ) fell just $10 \%$ below discrimination (chance level $=.50$ ) in the young adults and only $1 \%$ below discrimination in the middle-aged group. For the elderly, however, cued identification fell $50 \%$ below discrimination. Whereas the presence of cues would seem likely to counteract problems of retrieval from memory in both the young and the old, it seems to work better for the nonelderly. Cued identification, at least as performed here, stands to exaggerate differences from middle age to old age.

Cain et al. (in press) found that for real-world items, as opposed to simulants, free identification showed essentially no decline with age from young to middle-aged adulthood even though threshold increased. They concluded that the process of learning names of odors continues well into adulthood, even as sensitivity has begun to wane. Learning apparently overcomes the loss of sensitivity somewhat and keeps performance at identification propped up into middle age. This trend was not so apparent in the present study for free identification of the simulants, which were less well identified than real-world items, but the trend did seem true for cued identification. Just as cued identification seemed to exaggerate the effects of aging from middle age to old age, so it also seemed to diminish the effects of aging slightly from young adulthood to middle age (Figures 1 and 5).

When age and its attendant cognitive limitations are not an issue, cued identification yields performance surprisingly close to that obtained in discrimination. Hence, performance in the less time-consuming task of cued identification can serve to estimate that in the more time-consuming task of discrimination. For the elderly, cued identification may prove a somewhat weak substitute for a presumably less cognitively demanding task, such as absolute threshold or quality discrimination. This disparity may increase or decrease with how well the stimuli simulate real-world items. Conceivably, it might be smallest for natural stimuli where there is no need to "bend" qualities into the templates built up through decades of experience.

\section{REFERENCES}

CAIN, W. S. (1979). To know with the nose: Keys to odor identification. Science, 203, 467-470.

CAIN, W. S. (1989). Testing olfaction in a clinical setting. ENT Journal, 68, 316-328.
CaIN, W. S., \& GENT, J. F. (1986). Use of odor identification in clinical testing of olfaction. In H. L. Meiselman \& R. S. Rivlin (Eds.), Clinical measurement of taste and smell (pp. 170-186). New York: Macmillan.

Cain, W. S., Gent, J. F., Goodspeed, R. B., \& Leonard, G. (1988). Evaluation of olfactory dysfunction in the Connecticut Chemosensory Clinical Research Center. Laryngoscope, 98, 83-88.

CAIN, W. S., \& RABIN, M. D. (1989). Comparability of two tests of olfactory functioning. Chemical Senses, 14, 479-485.

CaIN, W. S., \& STEvens, J. C. (1989). Uniformity of olfactory loss in aging. Annals of the New York Academy of Sciences, 561, 29-38. Cain, W. S., Stevens, J. C., Nickou, C., Johnston, I., Gills, A., \& Garcia Medina, M. R. (in press). Life-span development of odor identification, learning, and olfactory sensitivity. International Journal of Behavioral Development.

Doty, R. L., Shaman, P., Applebaum, S. L., Giberson, R., SikorSKI, L., \& RosenberG, L. (1984a). Smell identification ability: Changes with age. Science, 226, 1441-1443.

Doty, R. L., Shaman, P., \& Dann, M. (1984b). Development of the University of Pennsylvania Smell Identification Test: A standardized micro-encapsulated test of olfactory functioning. Physiology \& Behavior, 32, 489-502.

Eskenazi, B., Cain, W. S., \& Friend, K. B. (1986). Exploration of olfactory aptitude. Bulletin of the Psychonomic Society, 24, 203-206.

Eskenazi, B., Cain, W. S., Novelly, R. A., \& Friend, K. B. (1983). Olfactory functioning in temporal lobectomy patients. Neuropsychologia, 21, 365-374.

Eskenazi, B., Cain, W. S., Novelly, R. A., \& Mattson, R. (1986). Odor perception in temporal lobe epilepsy patients with and without temporal lobectomy. Neuropsychologia, 24, 553-562.

Gescheider, G. A. (1984). Psychophysics: Method, theory, and application (2nd ed.). Hillsdale, NJ: Erlbaum.

Martinez, B., Cain, W. S., de Wijk, R. A., Spencer, D. D., Novelly, R., \& SASS, K. J. (1993). Olfactory functioning before and after temporal lobe resection for intractable seizures. Neuropsychology, 7, 351-363.

MuRPHY, C. (1983). Age-related effects on the threshold, psychophysical function, and pleasantness of menthol. Journal of Gerontology, 38, 217-222.

Pollack, I., \& Norman, D. A. (1964). A non-parametric analysis of recognition experiments. Psychonomic Science, 1, 125-126.

RABIN, M. D. (1988). Experience facilitates olfactory quality discrimination. Perception \& Psychophysics, 44, 532-540.

SChemper, T., Voss, S., \& CaIN, W. S. (1981). Odor identification in young and elderly persons: Sensory and cognitive limitations. Journal of Gerontology, 36, 446-452.

Schiffman, S. S., Moss, J., \& ERICkson, R. P. (1976). Thresholds of food odors in the elderly. Experimental Aging Research, 2, 389-398.

Stevens, J. C., \& Cain, W. S. (1985). Age-related deficiency in the perceived strength of six odorants. Chemical Senses, 10, 517-529.

Stevens, J. C., Cain, W. S., \& Demarque, A. (1990). Memory and identification of simulated odors in elderly and young persons. Bulletin of the Psychonomic Society, 28, 293-296.

WoOD, J. B., \& HARKINs, S. W. (1987). Effects of age, stimulus selection, and retrieval environment on odor identification. Journal of Gerontology, 42, 584-588.

ZatorRe, R. J., \& Jones-Gotman, M. (1991). Human olfactory discrimination after unilateral frontal or temporal lobectomy. Brain, 114, 71-84.

(Manuscript received November 23, 1992; revision accepted for publication December 19, 1993.) 